\title{
Le nettoyage des machines à traire les brebis dans la région de Roquefort. Adaptation du procédé semi-automatique, à l'eau chaude acidifiée, sur les machines à traire "système Casse"
}

\author{
par \\ M. GALZIN \\ Laboratoire de la Société des Caves - 12 - Roquefort (1)
}

\section{I. - Introduction}

Dans le rayon Roquefort, le nombre des machines à traire est en augmentation. Au $1^{\text {er }}$ janvier 1967 il y avait 214 machines «système Casse » en service, 501 au $1^{\text {er }}$ janvier 1971. Quant au nombre des machines tous modèles il est passé de 5 à 600 en 1967 à 900 . 1000 en 1971. Le fromage de Roquefort est fabriqué à partir de lait cru, peuplé d'une flore naturelle, en majorité lactique. Or, cette flore naturelle n'est pas du tout la même pour un lait, trait à la main, dans une bergerie, suivant la méthode traditionnelle et, pour un lait récolté à la machine, dans une salle de traite moderne, indépendante de la bergerie.

Le lait de brebis, cru, récolté à la machine pose deux problèmes différents :

- Le premier, est un problème de quantité de flore : la machine est souvent mal ou insuffisamment nettoyée. C'est chose courante en pays calcaire où l'eau est rare l'été ; le lait récolté est trop peuplé en germes, il est trop riche en flore et souvent contaminé par des coliformes. Un tel lait, (plus de $10^{7}$ germes au gramme) ne convient absolument pas à la fabrication normale du fromage de Roquefort. Richard et Auclair, dans leurs travaux ont bien

(1) Ce travail, a été réalisé au Laboratoire de la Société des Caves de Roquefort (Directeur : M. Bret) avec le concours de la laiterie expérimentale (MM. Assénat et Stoffel) du service des fermes expérimentales de la Société des Caves (Directeur : M. Bosc) et en particulier, avec la collaboration technique de MM. D. Soulas et L. Bonnefous, ainsi que du personnel technique des fermes du Casse et du Bousquet-Bas. Enfin, avec le concours du service technique de développement agricole (M. Ch. Delmas). 
éclairci cette question. Entre autre ils ont montré les progrès importants que l'on pouvait attendre de l'utilisation d'eau bouillante.

- Le deuxième problème, peut être plus particulier à Roquefort, est un problème de qualité de flore. Quand on atteint un certain niveau de qualité du lait, niveau que l'on peut situer à moins $10^{5}$ germes/g, il se pose au fabricant de fromage en cru, un nouveau problème, plus complexe. Ce qui devient important c'est la qualité de la flore, c'est-à-dire l'équilibre des groupes microbiens entre eux. Pour traiter de cette question, il nous faut décrire, le plus brièvement possible les essais effectués pendant 4 ans à la ferme du Casse, sur la bactériologie des laits récoltés à la machine.

\section{II. - Résultats des expérimentations faites à la ferme du Casse depuis 1967, sur la bactériologie des laits de machine à traire}

Le lait cru, avec sa flore naturelle, donne des fromages qui ont du goût. Mais, si cette flore est déséquilibrée ou trop abondante nous obtenons des fromages de basse qualité. C'est pourquoi, la Société des Caves a mené une politique vigoureuse de qualité du lait, surtout chez les producteurs possédant une machine à traire. Par la suite nous avons constaté une amélioration sensible de la qualité du fromage, qui suivait l'amélioration de la qualité du lait.

Pourtant, nous avons connu de sérieuses difficultés à la laiterie expérimentale du laboratoire quand il a fallu fabriquer du fromage de Roquefort avec des laits de machine, très propres, pauvres en germes. Il s'agissait très précisément du lait livré par la ferme du Casse. Nous ne pouvions obtenir du bon Roquefort à partir d'un lait de très haute qualité. La technique de nettoyage que nous faisions appliquer était très classique : rinçage, passage d'une lessive détergente, rinçage à l'eau tiède suivi d'une désinfection à l'eau javellisée, la dose courante utilisée est : $2 \mathrm{ml}$ d'eau de javel à $22^{\circ} \mathrm{Cl}$ pour 201 d'eau ; l'eau javellisée séjourne dans la machine entre les deux traites.

Depuis 1965, nous savions que, si on diminue, par un nettoyage rigoureux, la quantité de germes d'un lait de machine (jusqu'à 25 à 40000 germes au gramme) on ne retrouve pratiquement aucun germe lactique. L'essentiel de la population microbienne est constitué de microcoques et de germes de contamination (air, eau) que nous appellerons provisoirement : " alcalinisants " (2).

(2) Germes alcalinisants : sur milieu Plate Count Agar de Difco, additionné d'un indicateur de $\mathrm{pH}$ (pourpre de bromocrésol), ces germes donnent des colonies entourées d'un halo bleu, même si l'ensemble de la boîte a viré au jaune sous l'action acidifiante des bactéries lactiques. 
En 1966, nous confirmons ces observations. De plus, des essais de maturation du lait avec ou sans ferment exogène, nous montrent que ces laits de machine très propres sont très vite envahis par une flore de contamination très souvent alcalinisante (l'acidité du lait diminue au lieu d'augmenter) qui se développe en terrain libre par manque de flore lactique antagoniste.

En 1967, nous démarrons des essais de plus grande envergure, ils démontrent que :

1) L'eau javellisée qui séjourne dans la machine entre deux traites ne stérilise pas la machine, bien au contraire cette eau constitue un véritable bouillon de culture pour la flore alcalinisante de contamination.

2) L'eau de rinçage avant la traite, contamine également le lait, car elle contient davantage de germes que le lait récolté. Ceci provenait de la mauvaise qualité de l'eau de citerne utilisée. Ce problème a été résolu par l'installation d'une bougie filtrante.

3) Les ferments lactiques du commerce se développent mal dans un lait de ce type.

4) On constate avec ces laits très propres, que les tests classiques de fromagerie : réductase, lacto-fermentateur donnent des résultats incohérents.

5) Nous avons pu vérifier que ce lait ne contenait pas de résidus de Chlore ou de lessives pouvant expliquer le mauvais développement des ferments lactiques. Le lait a pourtant un pouvoir bactériostatique très net. On peut l'attribuer à la présence de lacténines (globulines) ou de leucocytes, ou plus simplement à l'implantation d'une flore antagoniste des bactéries lactiques.

A ce stade des recherches nous tirons les conclusions suivantes: la récolte du lait sur une machine bien nettoyée, conduit à supprimer toutes les causes traditionnelles et on pourrait dire « naturelles » d'ensemencement du lait. En conséquence de quoi, on obtient un lait de très faible teneur en germes, ces germes sont tous indésirables ou impropres à la fromagerie, ils proviennent pratiquement tous de contaminations.

A partir du mois de mai 1967, un système de stérilisation à la vapeur est installé sur une machine à traire à la ferme du Casse. Cette machine, stérilisée avant ou après chaque traite nous servira de témoin.

Les essais qui suivront vont montrer que la stérilisation à la vapeur donne des résultats bien supérieurs aux techniques de nettoyage, " détergent plus javel ", qui étaient la règle jusque-là. Cette observation est importante, car elle remet en cause l'hypothèse de départ : la machine à traire est moins bien nettoyée qu'on pouvait le supposer. On peut obtenir un lait cru contenant 5 à 10000 germes au gramme contre 40 à 50000 germes dans le meilleur des cas pour la technique à l'eau javellisée. 


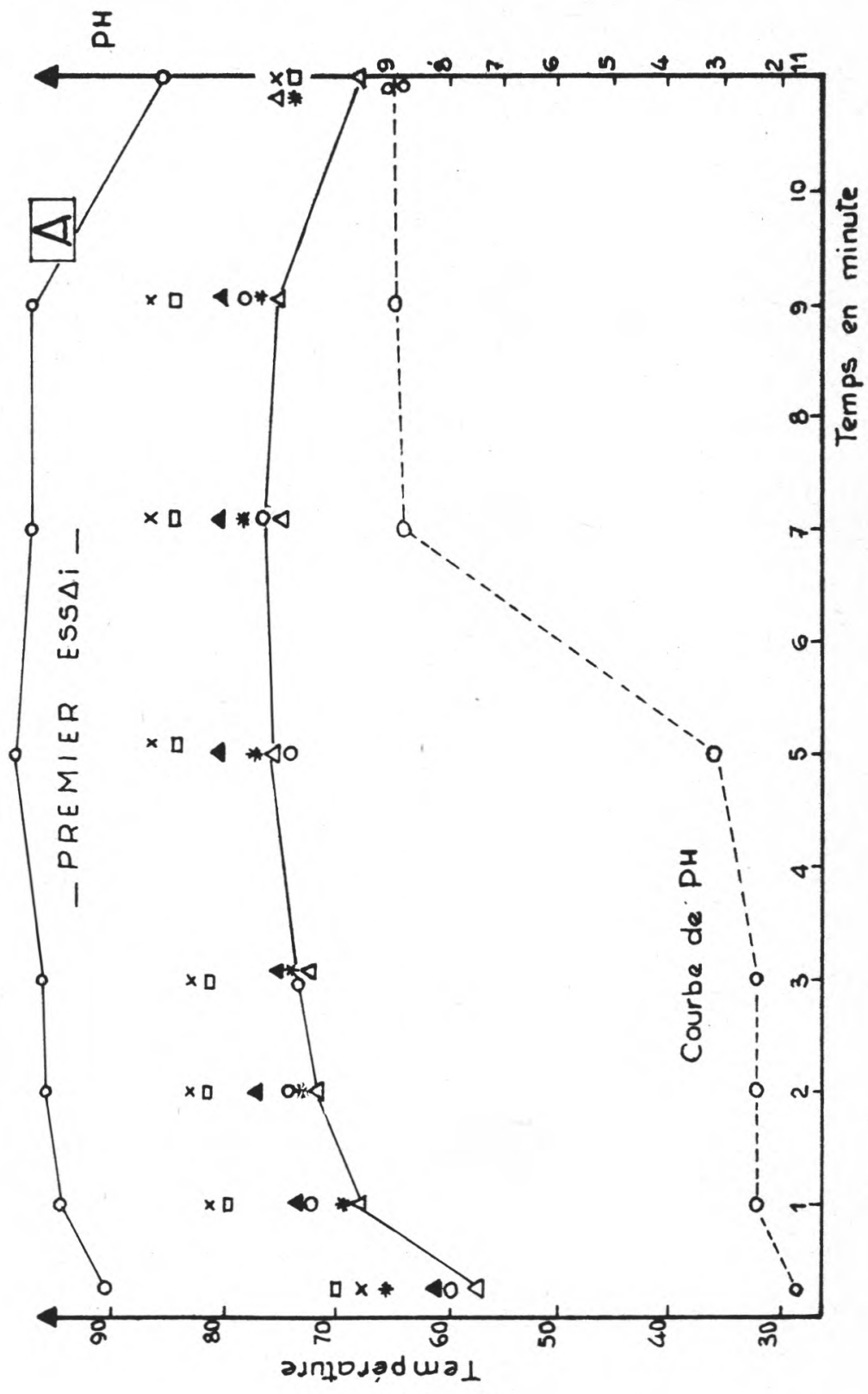

graphique $1 \mathrm{~A}$

Enregistrement des températures et du $\mathrm{pH}$ de l'eau chaude acidulée, en différents points de la machine, pendant l'opération de nettoyage.

- Abcisse : temps en minutes.

- ordonnée gauche : température en degré Celsius.

- Ordonnée droite : $\mathrm{pH}$ en unités $\mathrm{pH}$.

- Légende des courbes :

A premier essai, première journée. 
I

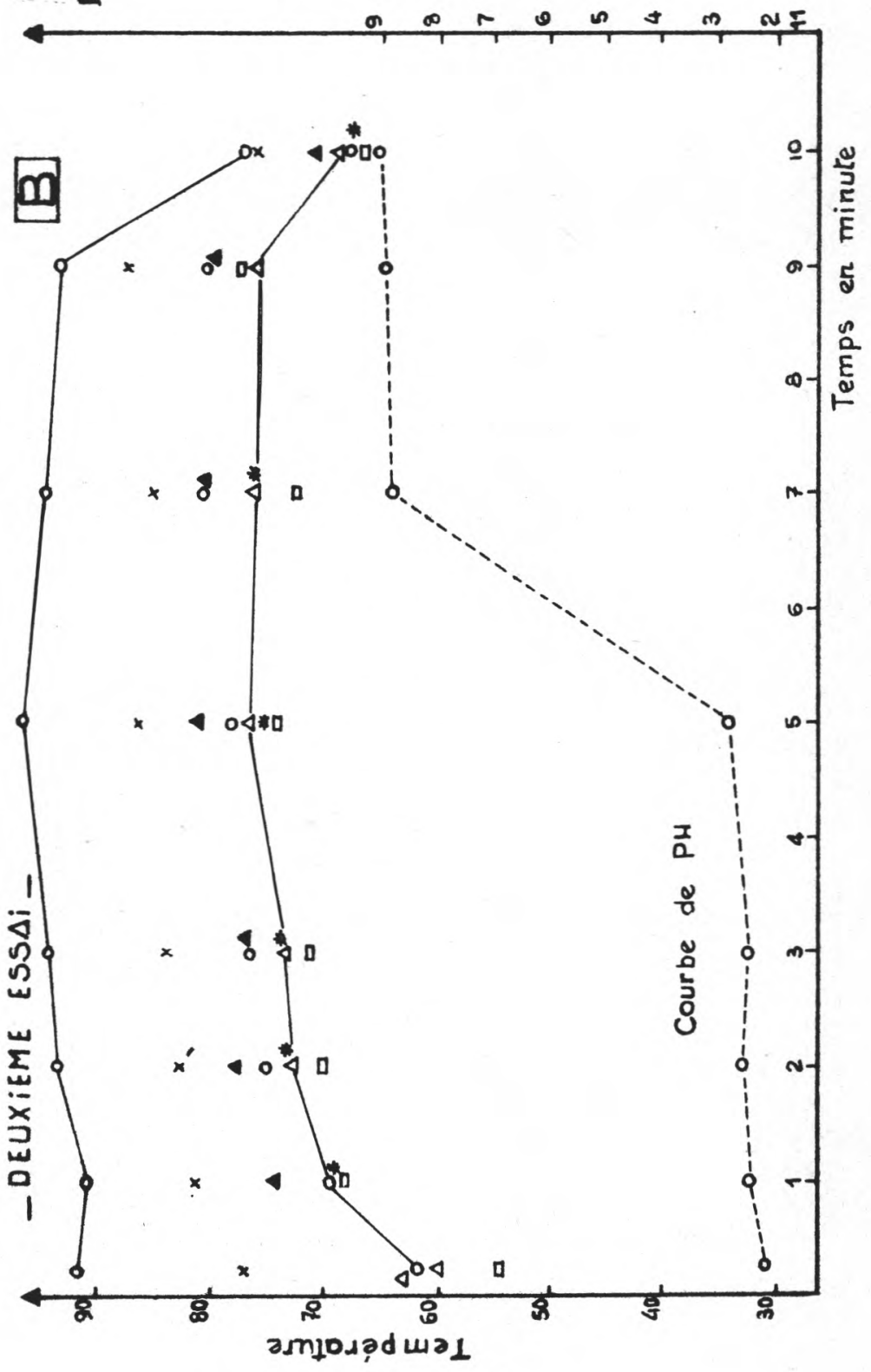

graphique $1 B$

B deuxième essai, deuxième journée. entrée dans la machine, départ. premier gobelet (début fosse). deuxième gobelet (milieu fosse). troisième gobelet (fin de fosse). ligne de transfert. boucle en fin de ligne de transfert. sortie machine. courbe de $\mathrm{pH}$. 
Avant d'aller plus loin, nous mettons sur pied un essai pour vérifier si le nettoyage du pis des brebis apporte une amélioration. La réponse est non. Cette source de contamination paraît assez négligeable : les brebis servant aux essais ont les pis très propres. Il faut préciser que la paille est abondante sur les litières.

En 1968, une série de 4 essais fut réalisée :

1) Premier essai : durée 7 semaines en janvier, février, mars 1968.

Il s'agissait d'étudier les trois origines pcssibles des contaminations : la machine à traire, les brebis (flore intramammaire), l'ambiance (air - poussières).

Cet essai apporte les réponses suivantes :

1.1. Pendant la traite, l'air, les poussières aspirées par les gobelets, apportent relativement peu de germes.

1.2. La flore de contamination est très fluctuante en quantité et en nature des germes. Toutefois, elle comporte comme base très régulière, comme constante, une quantité importante de microcoques. Ils sont probablement d'origine intramammaire. Ils représentent environ $1 / 3$ de la flore totale. A titre de comparaison, on trouve dans un lait courant, au maximum 1 à 5 p. 100 de microcoques.

1.3. La stérilisation à la vapeur de la machine permet de diminuer de 2 à 3 fois la quantité de germes totaux. Elle permet surtout de diminuer, dans la proportion de 20 à 1 le nombre des alcalinisants.

1.4. Un lot de brebis ayant eu des mammites est trait à part et le lait caillé à part. On peut vérifier que la flore totale est deux fois plus élevée que la normale et que cela donne des fromages de très mauvaise qualité.

1.5. Nous avons même ensemencé à plusieurs reprises la salle de traite avec des ferments lactiques en aérosols. Les résultats n'ont pas été bien nets.

1.6. La conclusion d'ensemble que l'on a pu tirer est la suivante : dans l'ensemencement du lait récolté à la machine, l'ambiance, l'environnement semblent jouer un rôle mineur. La grosse majorité des germes provient de la machine. Le nettoyage des circuits, des manchons, des gobelets, des caoutchoucs est essentiel. Le rôle des mammites latentes semble être assez important.

2) Dans les deuxième, troisième et quatrième essais de 1968, nous avons creusé la question des mammites latentes et confirmé les résultats déjà obtenus.

Nous avons analysé séparément le lait de 335 brebis. Les brebis sont séparées en deux lots. Un lot appelé un peu improprement « germfree " qui donne un lait contenant moins de 100 germes $/ \mathrm{ml}$ et un lot témoin dont le lait contient une moyenne de 20000 germes $/ \mathrm{ml}$. 
Ces deux lots sont traits séparément et successivement sur la machine stérilisée à la vapeur de façon à éliminer l'apport de germes par la machine. Le lait récolté est analysé et caillé à part pendant plusieurs jours.

Les conclusions de cet essai sont très claires :

2.1. La qualité de la flore (teneur en microcoques) a une influence certaine sur la texture du caillé et sur le raffermissement du grain pendant les brassages.

2.2. On n'a pas constaté de gros écarts entre la teneur en microcoques du lait témoin et celle du lait essai. Cela semble indiquer que la flore intramammaire (mammites latentes) ne joue pas un rôle si important qu'on le pensait, ou bien, le lot de brebis, en principe riche en mammites latentes, était moins riche que prévu et n'apportait dans le lait qu'une flore banale. Nous pensions que les mammites latentes pouvaient être à l'origine de toutes nos difficultés. Cet essai démontre que les mammites certes jouent un rôle, mais que ce n'est pas le fond du problème.

2.3. Nous avons obtenu, au cours des essais de caillage à part des fromages d'excellente qualité. Ces fromages avaient été fabriqués à partir d'un lait cru ne contenant que 4400 germes totaux/g et moins de 10 coliformes/g. Le fromage s'est très bien affiné. Il a été dégusté 12 mois après : sa conservation et son goût étaient excellents. Il faut préciser que nous avions ajouté au lait un mélange de plu. sieurs espèces différentes de bactéries, isolées à partir de lait cru de brebis et sélectionnées. Nous savions maintenant qu'il était possible d'obtenir du Roquefort à partir d'un lait très pauvre en germes, récolté à la machine à traire.

Essai 1969 : Pendant l'intercampagne (été 1968) le système de nettoyage à l'eau chaude acidifiée, est installé sur la deuxième machine à traire de la ferme du Casse.

Nous allons étudier ce système de nettoyage en le comparant à la première machine à traire, stérilisée à la vapeur d'eau, et servant de témoin.

\section{III. - Le nettoyage des machines à traire par le procédé à l'eau chaude acidifiée}

C'est en 1968, que nous avons eu connaissance de la technique de nettoyage à l'eau chaude acidifiée. Elle était alors expérimentée en Angleterre au Centre de Recherches Laitières de Reading. Les avantages de la méthode paraissaient nombreux, il restait à vérifier la qualité dú nettoyage obtenu. 


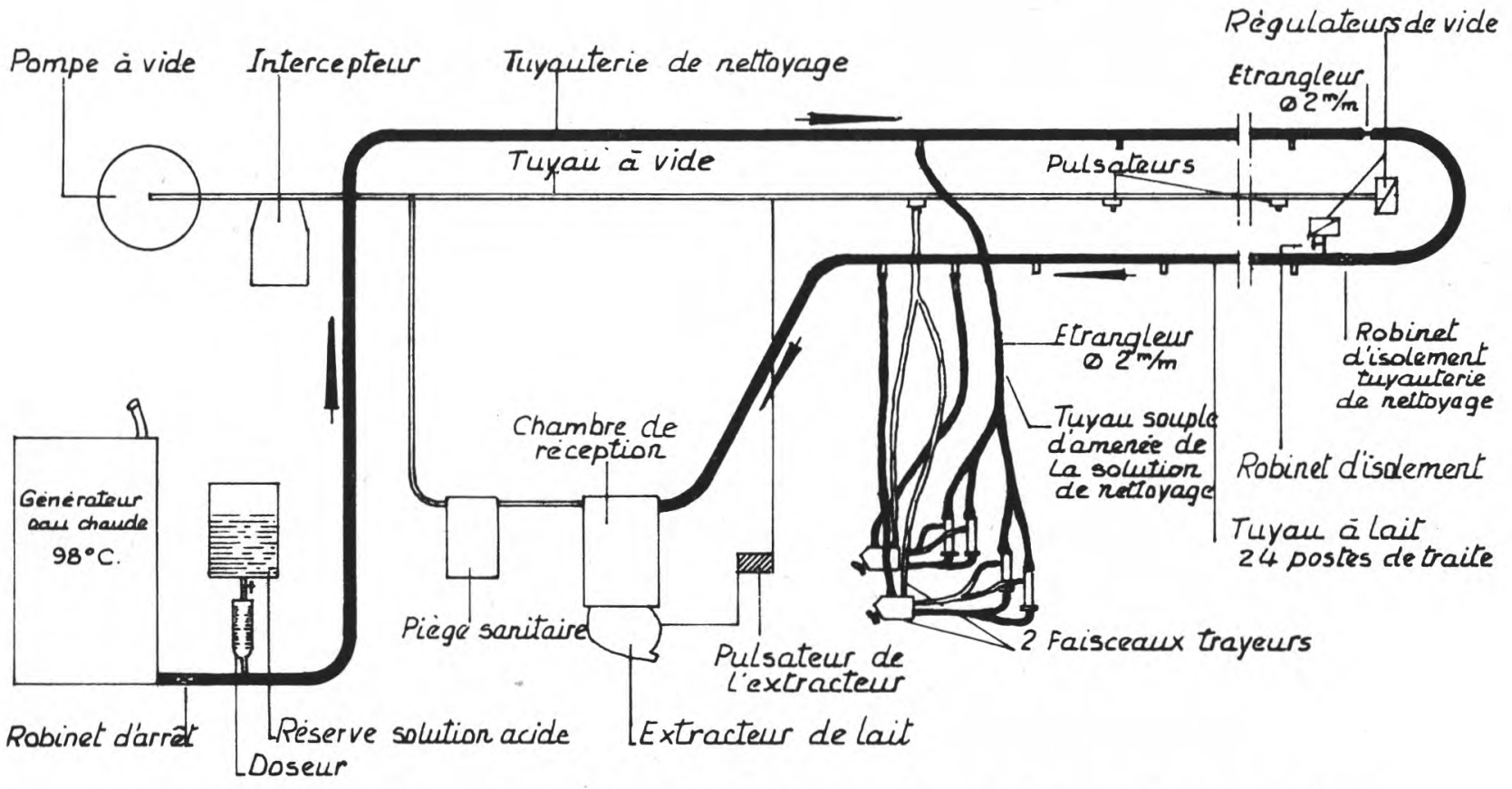

\section{TRAITE MECAMIQUE SYSTEME CASSE}

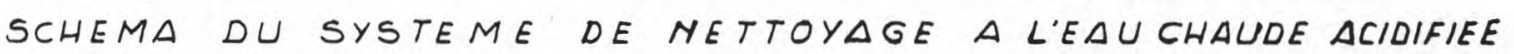


III. - 1 : MATÉRIEL ET MÉTHODES

\section{III. - 1.1. Présentation du dispositif expérimental}

Les essais ont été conduits à la ferme expérimentale du Casse. Nous l'avons vu précédemment, nous disposions de deux fosses de traite à 48 places, modèle Alfa-Laval "système Casse " à double pose (3).

\subsubsection{Machine témoin}

Sur la première fosse, la machine L1 est équipée d'un système de stérilisation à la vapeur d'eau. C'est la machine de référence, la machine témoin. Il nous a fallu pour cela, remplacer la tuyauterie plexigum par une tuyauterie inox (ou de verre pyrex). Le plexigum se déforme aux températures de stérilisation. Avant la stérilisation, qui dure de 30 à $40 \mathrm{mn}$; la machine est nettoyée par une lessive détergente non-chlorée.

\subsubsection{Machine essai}

Sur la deuxième fosse, la machine L2, est équipée du système de nettoyage à l'eau chaude acidifiée (4).

La source d'eau chaude est un chauffe-eau électrique de $120 \mathrm{I}$. Il fonctionne à pression atmosphérique, il est équipé d'un thermostat, l'eau est portée à $98^{\circ} \mathrm{C}$.

La circulation de l'eau chaude est assurée par le vide de la machine à traire, l'eau est aspirée dans le circuit nettoyage, puis elle passe à travers les gobelets et retourne par la canalisation de transfert du lait.

Chaque prise d'eau sur la canalisation de nettoyage alimente 4 gobelets et ceci au moyen de 4 tuyaux de plastique souple, terminés par 4 tétines d'aspiration. Pour régulariser le débit, chaque tuyau plastique est obstrué par un gicleur de réduction. L'évacuation de l'eau chaude sans rupture du vide se fait par une chambre d'écluse.

Une fois les gobelets branchés sur les tétines d'aspiration et la chambre d'écluse en place, le système est prêt à fonctionner en circuit ouvert. En effet, il faut bien noter qu'il n'y a pas circulation d'eau en circuit fermé. L'eau chaude admise à l'entrée de la machine est évacuée à la sortie.

(3) Pour plus de précisions, nous renvoyons aux deux articles suivants :

- Société Anonyme des Caves et des Producteurs Réunis de Roquefort : Traite mécanique des brebis "système Casse ". $2^{\mathrm{e}}$ édition 1967.

- Bosc (J.) : Progrès récents des problèmes de la traite mécanique des brebis. Ann. de la Nutrition et de l'Alimentation, 1966, vol. XX, $\mathrm{n}^{\circ} 6$, p. $193-230$.

(4) Nous tenons à remercier la firme Alfa-Laval pour sa contribution technique ainsi que pour la fourniture du matériel de nettoyage et du générateur d'eau chaude. 
On prépare une solution d'acide sulfamique à $1 \mathrm{~kg}$ d'acide en cristaux pour 51 d'eau. On verse à chaque nettoyage 1 à 1,51 de cette solution acide dans un entonnoir doseur disposé juste avant la vanne d'admission de l'eau chaude. Au moment où on ouvre la vanne l'acide est aspiré régulièrement en même temps que l'eau chaude. Les anglais ont utilisé aussi l'acide nitrique.

\subsubsection{Machines de référence}

Pour avoir de solides points de comparaison, nous avons suivi pendant la période expérimentale, trois autres machines à traire.

Une machine à traire appelée L4, installée à la ferme du Bousquet. C'est la deuxième ferme expérimentale de la Société des Caves. C'est une installation Alfa-Laval « système Casse » semblable à L1 et L2, mais avec 24 postes au lieu de 48 .

La technique de nettoyage est un peu particulière :

- Rinçage à l'eau froide immédiatement après la traite.

- Passage de lessive détergente désinfectante non moussante, avec une eau très chaude $\left(70-80^{\circ} \mathrm{C}\right)$. Temps de contact 10 à $15 \mathrm{mn}$.

- Rinçage à l'eau chaude.

- La machine est égouttée entre deux traites. On ne laisse plus séjourner d'eau javellisée dans les circuits entre les deux traites. tant.

- Chaque semaine ; détartrage avec un produit acide désincrus-

Deux machines à traire appelées $\mathrm{V}$ et $\mathrm{R}$ également de marque Alfa-Laval « système Casse " installées chez deux producteurs de lait de brebis de la région de Roquefort. Ces deux producteurs peuvent être considérés comme des producteurs-types, moyens de cette zone.

Leur technique de nettoyage est classique :

- Rinçage à l'eau froide.

- Passage de lessive avec eau chaude.

- Rinçage à l'eau chaude.

- Passage d'eau froide javellisée.

- Rinçage à l'eau froide.

- Démontage complet et détartrage une fois par mois environ.

\section{III. - 1.2. Prélèvements et analyses}

La mesure du $\mathrm{pH}$ de l'eau est faite à l'aide d'un pHmètre Tacussel à lecture numérique (ISIS 4000).

Les analyses bactériologiques du lait et des eaux sont effectuées pour les germes totaux sur milieu Plate Count Agar (Difco $\mathrm{n}^{\circ} 479$ ) additionné de pourpre de bromocrésol à 0,02 p. 100 (concentration finale) après incubation de $48 \mathrm{~h}$ à $25^{\circ} \mathrm{C}$. Les staphylocoques et les microcoques sont dénombrés sur milieu de Chapman (Mannitol Salt Agar de Difco $\mathrm{n}^{\circ} 30$ ) après incubation $72 \mathrm{~h}$ à $30^{\circ} \mathrm{C}$. 
Nous avons réalisé trois sortes d'essais :

Dans une première série d'essais nous étudions la technologie de cette méthode de nettoyage.

Ensuite, nous vérifions, par des prélèvements et des analyses bactériologiques, l'efficacité du nettoyage.

$\mathrm{Et}$, enfin nous analysons les laits récoltés sur les 5 machines que l'on compare. Cela, afin de vérifier le niveau de qualité bactériologique que l'on peut atteindre à partir de chaque type de nettoyage.

\section{III. - 2 : Technologie du NetToyage a L'EAU Chaude acidifiée}

La température de l'eau chaude acidifiée est enregistrée en différents points de la machine. Nous suivons la température de l'eau chaude depuis l'admission jusqu'à l'évacuation. Sept thermomètres sont placés le long du circuit de nettoyage. Toutes les minutes, nous enregistrons les températures indiquées.

Dans le même temps, l'eau de nettoyage est récupérée toutes les minutes et le $\mathrm{pH}$ est mesuré.

Ces mesures sont réalisées pendant $3 \mathrm{j}$ consécutifs et les résultats exprimés sous forme de graphique (graphique 1: A et B).

\section{Discussion}

La durée totale du nettoyage proprement dit, n'excède par $11 \mathrm{mn}$.

Pendant $9 \mathrm{mn}$, l'eau entre dans la machine à une température de l'ordre de $93^{\circ} \mathrm{C}$ à $94^{\circ} \mathrm{C}$. A la sortie, l'eau est à une température supérieure à $70^{\circ} \mathrm{C}$ pendant $8 \mathrm{mn}$. Cela semble suffisant pour assurer une destruction convenable des germes.

Dans la boucle de retour, en fin de ligne de transfert, la température enregistrée est la même qu'à la sortie de la machine. Ce sont les deux points critiques, car la température y est la plus basse.

Les températures relevées dans les gobelets trayeurs varient suivant que le gobelet est en début, au milieu ou en fin de circuit. Mais on constate certains circuits préférentiels : par exemple le deuxième jour, le deuxième gobelet (en milieu de fosse) est resté à une température inférieure à celle des autres gobelets. Dans la plupart des cas, une prise d'air est à l'origine de ce phénomène.

Pendant $5 \mathrm{mn}$, l'eau de lavage circule dans la machine à un $\mathrm{pH}$ compris entre 2 et 3 . C'est pendant cette période que l'effet bactéricide est le plus marqué ; il y a action conjuguée de la température et $\mathrm{du} \mathrm{pH}$ acide.

Ensuite, pendant 5 à $6 \mathrm{mn}$, l'eau chaude circule sans acide, le $\mathrm{pH}$ étant remonté aux environs de 8 à 9 . On peut modifier la durée de la phase acide en faisant varier la quantité de solution acide injectée à l'entrée du circuit de nettoyage. 
TABLEAU 1. - Vérification de l'action bactéricide de l'eau chaude acidifiée

\begin{tabular}{|c|c|c|c|c|c|c|c|}
\hline & & \multicolumn{2}{|c|}{ Premier jour } & \multicolumn{2}{|c|}{ Deuxième jour } & \multicolumn{2}{|c|}{ Troisième jour } \\
\hline & & $\begin{array}{l}\text { Flore } \\
\text { totale }\end{array}$ & Microcoques & $\begin{array}{l}\text { Flore } \\
\text { totale }\end{array}$ & Microcoques & $\begin{array}{l}\text { Flore } \\
\text { totale }\end{array}$ & Microcoques \\
\hline $\begin{array}{l}\text { Début nettoyage } \\
1 \mathrm{mn} \\
2 \mathrm{mn} \\
3 \mathrm{mn} \\
5 \mathrm{mn} \\
7 \mathrm{mn} \\
9 \mathrm{mn} \\
10 \mathrm{mn} \\
11 \mathrm{mn} \\
12 \mathrm{mn} \\
\text { Frottis grille } \\
\text { Eau résiduaire } \\
\text { dans les } \\
\text { manchons } \\
\text { Frottis } \\
\text { sur les } \\
\text { manchons }\end{array}$ & $\begin{array}{l}1 \\
2 \\
3 \\
1 \\
2 \\
3\end{array}$ & $\begin{array}{r}210 \\
0 \\
0 \\
0 \\
0 \\
2 \\
0 \\
0 \\
0 \\
5 \\
20 \\
<10 \\
\\
\\
<10 \\
<10 \\
200\end{array}$ & $\begin{array}{r}17 \\
0 \\
0 \\
1 \\
1 \\
0 \\
0 \\
0 \\
0 \\
\\
8 \\
3 \\
\\
\\
\\
\\
0 \\
1 \\
43\end{array}$ & $\begin{array}{r}60 \\
0 \\
0 \\
1 \\
2 \\
0 \\
0 \\
\times \quad 0 \\
\\
400 \\
10 \\
<10 \\
30 \\
<10 \\
20\end{array}$ & $\begin{array}{r}60 \\
0 \\
0 \\
0 \\
0 \\
0 \\
0 \\
0 \\
\\
\\
\\
10 \\
35 \\
2 \\
6 \\
1 \\
3\end{array}$ & $\begin{array}{r}80 \\
4 \\
10 \\
1 \\
0 \\
1 \\
0 \\
1 \\
0 \\
300 \\
60 \\
50 \\
<10\end{array}$ & $\begin{array}{r}19 \\
0 \\
2 \\
0 \\
0 \\
0 \\
0 \\
0 \\
0 \\
<10 \\
3 \\
4 \\
5\end{array}$ \\
\hline
\end{tabular}


TABLEAU 2. - Contrôle de l'efficacité du nettoyage

Analyses bactériologiques sur le lait stérile, utilisé pour rincer les machines L1 et L2 après nettoyage

\begin{tabular}{|c|c|c|c|c|c|c|c|}
\hline \multirow{2}{*}{ Machine } & \multirow{2}{*}{ Prélèvement } & \multicolumn{2}{|c|}{ Premier jour } & \multicolumn{2}{|c|}{ Deuxième jour } & \multicolumn{2}{|c|}{ Troisième jour } \\
\hline & & Flore totale & Microcoques & Flore totale & Microcoques & Flore totale & Microcoques \\
\hline L 1 & $\begin{array}{l}1 \\
2 \\
3\end{array}$ & $\begin{array}{r}610 \\
110 \\
10\end{array}$ & $\begin{array}{l}<10 \\
<10 \\
<10\end{array}$ & $\begin{array}{r}380 \\
80 \\
<10\end{array}$ & $\begin{array}{r}10 \\
10 \\
<10\end{array}$ & $\begin{array}{l}10 \\
60 \\
20\end{array}$ & $\begin{array}{l}<10 \\
<10 \\
10\end{array}$ \\
\hline L 2 & $\begin{array}{l}1 \\
2 \\
3\end{array}$ & $\begin{array}{r}14000 \\
60000 \\
9000\end{array}$ & $\begin{array}{r}30 \\
<10 \\
90\end{array}$ & $\begin{array}{r}11500 \\
8200 \\
1500\end{array}$ & $\begin{array}{r}10 \\
20 \\
<10\end{array}$ & $\begin{array}{l}730 \\
230 \\
530\end{array}$ & $\begin{array}{r}<10 \\
<10 \\
20\end{array}$ \\
\hline
\end{tabular}


III. - 3 : VÉRIFICATION DE L'ACTION BACTÉRICIDE DE L'EAU CHAUDE ACIDIFIÉE

Pour cela, nous avons prélevé, toutes les minutes, une fraction de l'eau de lavage.

Nous avons également récupéré, à la fin du nettoyage, l'eau de lavage qui reste, qui persiste dans les manchons et les gobelets d'une traite à l'autre.

Pour être complets, nous avons également analysé des frottis sur manchons ainsi que des raclages de dépôt sur les griffes à lait.

Les résultats sont donnés sur le tableau 1.

\section{Discussion}

L'eau chaude acidifiée qui sort de la machine ne contient pratiquement aucun germe vivant.

Les frottis et les prélèvements d'eau séjournant dans la machine, donnent des résultats irréguliers, mais très satisfaisants ; il apparaît cependant que sur les parois des griffes et les manchons il reste quelques germes qui ont résisté au traitement.

En 1968, nous avions effectué à peu près les mêmes contrôles sur cette machine L2. Elle était nettoyée suivant la technique traditionnelle : lessive plus eau tiède, suivie d'un rinçage à l'eau javellisée. Nous avions trouvé que les résidus de solution désinfectante javellisée, qui séjournaient dans la machine entre deux traites, étaient en fait un véritable bouillon de culture, ayant un pouvoir sélectif en faveur des germes alcalinisants, très préjudiciables à la qualité du lait.

\section{III. - 4 : MEsure de L'efFicacité dE NETtoyage}

La machine Essai L2 et la machine Témoin L1 sont salies artificiellement, puis nettoyées. On vérifie l'efficacité du nettoyage en opérant un rinçage de la machine après le nettoyage, avec du lait stérilisé.

Dans les deux machines, on aspire à partir de trois gobelets trayeurs, un lait très contaminé. On choisit un gobelet en début, en milieu et en fin de ligne.

Les deux machines sont ensuite nettoyées suivant la technique habituelle (L1 stérilisation à la vapeur, L2 eau chaude acidifiée).

On rince la machine après le nettoyage avec du lait écrémé stérile. L'aspiration de ce lait stérile est effectuée à partir des trois gobelets trayeurs choisis ci-dessus. A la sortie de la machine on prélève trois fractions de ce lait de rinçage, qui correspondent aux trois gobelets par où se fait successivement l'aspiration.

Le contrôle est renouvelé $3 \mathrm{j}$ de suite.

Les résultats apparaissent dans le tableau 2. 


\begin{tabular}{|c|c|c|c|c|c|}
\hline Machines & \multicolumn{3}{|c|}{ Type } & Origine & Nettoyage \\
\hline L1 Témoin & Cass & ost & uble pose & Ferme & Stérilisation vapeur \\
\hline L2 Essai & $»$ & 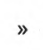 & $»$ & expérimentale & Eau chaude acidifiante \\
\hline L4 & Cass & oos & uble pose & Société des Caves & Méthode classique \\
\hline références & $»$ & $»$ & $»$ & Deux producteurs & $"$ \\
\hline $\mathrm{V}$ & $»$ & $»$ & " & brebis & $»$ \\
\hline
\end{tabular}




\section{Discussion}

En ce qui concerne les microcoques et les staphylocoques, les performances des deux machines sont comparables. Pour la flore totale, la machine Témoin L1 est supérieure à la machine Essai L2.

On constate une amélioration du $1^{\text {er }}$ au $3^{\text {me }}$ jour.

Les performances de L2 sont très convenables le $3^{\text {me }}$ jour. Les résultats montrent que certains gobelets sont mieux nettoyés que d'autres, cela est certainement dû à l'imperfection du matériel expérimental nouveau.

On ne peut pas dire que le nettoyage à l'eau chaude acidifiée corresponde à une stérilisation complète de la machine. Cependant les résultats obtenus paraissent intéressants.

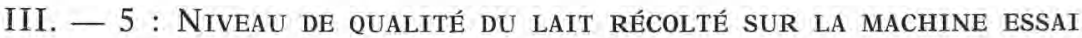

Afin de s'assurer de l'efficacité du nettoyage à l'eau chaude acidifiée, nous avons comparé pendant $15 \mathrm{j}$ les niveaux de flore de cinq machines à traire différentes. Les traites du soir et du matin étaient prélevées séparément.

Résultats sur les graphiques 2 et 3 et sur le tableau 3.

\section{Discussion}

- Graphique 2 : Le lait récolté sur L1 et L2 est de très bonne qualité bactériologique. Les performances des deux machines sont très proches et régulières. Le niveau de la flore se situe entre $10^{4}$ et $10^{5}$ germes $/ g$ de lait. On peut noter que la flore totale est dans ces deux machines constituée en majorité de microcoques.

Les trois autres machines donnent des résultats moins bons, le nettoyage est certainement moins efficace. La teneur en germes varie beaucoup entre la traite du matin et la traite du soir, elle varie également d'un jour à l'autre.

Niveau moyen : L4 $10^{5}$ à $10^{6}$ germes/g.

$$
\begin{aligned}
& \mathrm{R} \text { » " } \\
& \mathrm{V} 10^{6} \text { à } 10^{8} \text { germes } / \mathrm{g} \text {. }
\end{aligned}
$$

- Graphique 3 : Pour les microcoques, staphylocoques, les différences sont moins marquées. En effet, on admet que dans un lait récolté dans une machine à traire, nettoyée convenablement, la majeure partie des microcoques sont d'origine intra-mammaire.

- Tableau 3 : Il donne la moyenne des teneurs en germes totaux et microcoques pour $15 \mathrm{j}$ et pour les cinq machines à traire.

Il fait apparaître les écarts dus aux techniques de nettoyage.

L1 et L2 sont comparables. Traites du soir et traites du matin ont à peu près les mêmes teneurs en germes. On peut en conclure que l'apport de germes dans le lait, provenant de la machine est pratiquement réduit à néant. 
TABLEAU 3

Moyennes des teneurs en germes totaux et microcoques pour les cinq machines à traire et pendant $15 \mathrm{j}$

\begin{tabular}{|c|c|c|c|c|}
\hline \multicolumn{5}{|c|}{ Moyennes } \\
\hline & \multicolumn{2}{|c|}{ Flore totale } & \multicolumn{2}{|c|}{ Microcoques } \\
\hline & matin & soir & matin & soir \\
\hline $\mathrm{L} 1$ & 53000 & 55000 & 28000 & 22500 \\
\hline $\mathrm{L} 2$ & 56000 & 58500 & 29500 & 22000 \\
\hline L4 & 178000 & 6800000 & 63000 & 52000 \\
\hline $\mathrm{R}$ & 115000 & 6100000 & 33000 & 35000 \\
\hline $\mathrm{V}$ & 3900000 & 70900000 & 124000 & 348000 \\
\hline
\end{tabular}




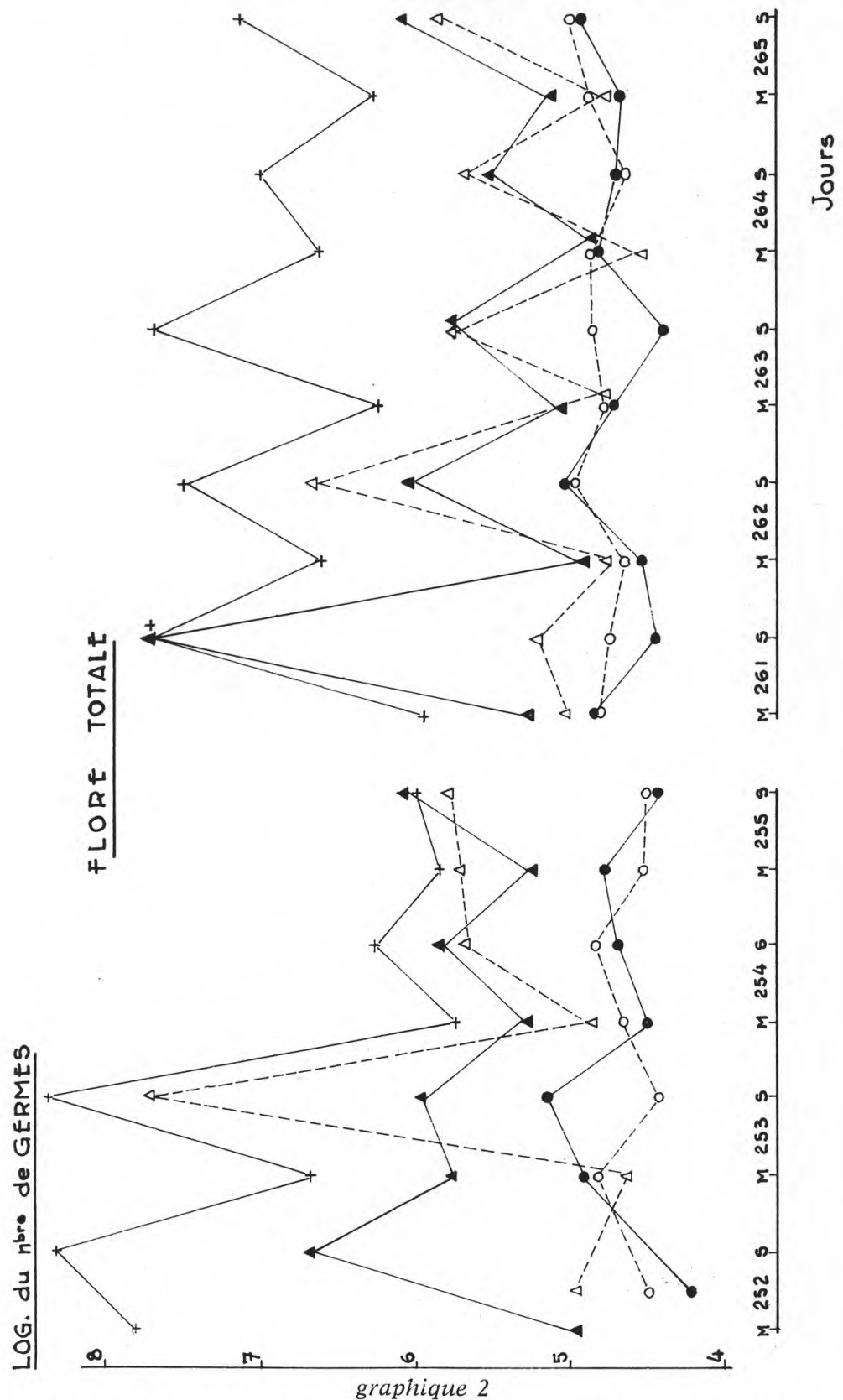

Niveau de flore des cinq machines, mise en comparaison pendant 2 semaines.

- Abcisse : chaque jour est indiqué par le "quantième " exemple : 252 semaines $252^{\text {me }}$ jour. $M$. et $\mathrm{S}$ indiquent les analyses sur traite du matin ou sur traite du soir.

- Ordonnée : logarithme du nombre de germes.

- Légende des courbes :

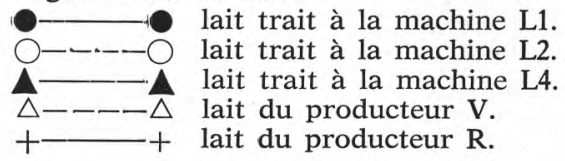



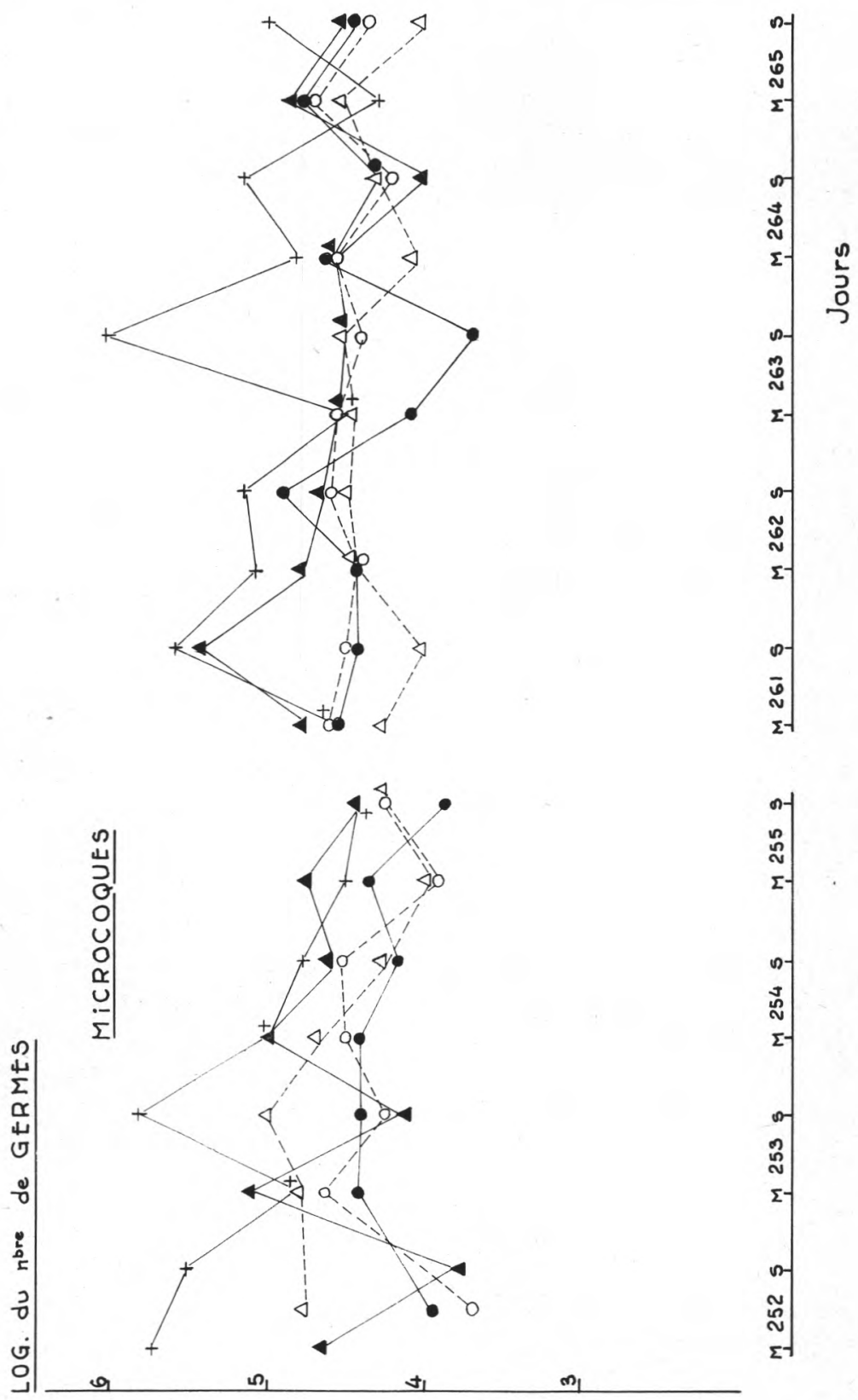

graphique 3

Niveau des microcoques staphylocoques pour les cinq machines, mises en comparaison pendant 2 semaines.

- Abcisse : idem ci-dessus.

- Ordonnée : idem ci-dessus.

- Légende : idem ci-dessus. 
Les techniques de nettoyage L1 et L2 suppriment pratiquement la contamination du lait par la machine.

Pour les trois autres machines, on constate des écarts importants entre traite du matin et traite du soir. Les microcoques font exception. Les niveaux de flore sont nettement supérieurs, surtout pour la machine $\mathrm{V}$.

D'après ce que nous connaissons des performances du nettoyage classique, on peut admettre que les machines L4 et $\mathrm{R}$ sont nettoyées convenablement. La machine $\mathrm{V}$ est certainement très mal nettoyée.

\section{IV. - Conclusion}

Les résultats du nettoyage à l'eau chaude acidifiée sont très supérieurs aux procédés actuellement utilisés. Sur le plan teneur en germes du lait, cette technique donne des résultats très remarquables. Si l'on tient compte de la qualité des germes ou de l'équilibre des groupes microbiens entre eux, on constate que l'on obtient un lait dont 50 p. 100 environ des germes totaux sont des microcoques. Dans un lait cru moyen, courant, le taux de microcoques est d'environ 1 p. 100. Il est évident qu'il faut tenir compte de ce fait dans la fabrication de fromages à partir de lait cru.

Cette technique de nettoyage est utilisée régulièrement au Casse depuis 1969 sur une machine, depuis 1970 sur les deux machines et quatre installations ont fonctionné chaque jour pendant la campagne 1971.

Nous commençons à avoir un peu de recul et il nous est possible d'affirmer que les résultats sont excellents. Nous avons fait des progrès importants dans la récolte des laits crus fromagers à la machine à traire.

Il est coûteux de généraliser le procédé aux machines déjà en place, car il est nécessaire d'installer des canalisations inox ou pyrex à la place du plexigum, mais nous pensons que toutes les installations neuves qui seront installées dans l'avenir devraient pouvoir bénéficier de ce procédé semi-automatique de nettoyage.

Par rapport au système classique actuellement en vigueur, et pour une installation 48 stalles comme au Casse, on peut faire la comparaison suivante :

- il est plus rapide : $15 \mathrm{mn}$ avec une personne au lieu de $25 \mathrm{mn}$ avec deux personnes,

- il est moins pénible : on ne porte plus de seaux de lessive jusqu'aux gobelets,

- on réalise une économie de produits détergents,

- on évite un détartrage hebdomadaire et la qualité du nettoyage est supérieure. 


\section{Rés u mé}

Le nettoyage des machines à traire les brebis dans la région de Roquefort. Adaptation du procédé semi-automatique, à l'eau chaude acidifiée, sur les machines à traire "Système Casse ".

Le fromage de Roquefort est fabriqué avec du lait cru de brebis. Il est donc important que le nettoyage des machines à traire les brebis soit efficace ; d'autant plus que leur nombre augmente chaque année.

Dans une première partie, nous passons en revue toutes les expérimentations, faites à la ferme expérimentale de la Société des Caves de Roquefort, au Casse.

Depuis 1967 jusqu'à 1970, les essais montrent les relations qui existent entre la qualité du lait cru et les techniques de nettoyage des machines, la qualité des eaux de rinçage, l'existence de mammites latentes, le nettoyage des trayons, l'atmosphère de la salle de traite, etc.

En 1968, une adaptation du procédé à l'eau chaude acidifiée (expérimentée au N.I.R.D. England) est installée sur une machine système Casse. Nous décrivons les essais de nettoyage, les mesures de l'efficacite du nettoyage et les résultats sur la qualité du lait.

Cette méthode est très bien adaptée et très efficace. Elle se développe depuis. Le lait récolté sur quatre machines à traire, nettoyées selon ce procédé est suivi depuis 1969. Le niveau de qualité est excellent.

Le procédé est de plus, très simple, pratique et trois fois plus rapide que les méthodes de nettoyage habituelles.

\section{S u m m a ry}

Cleaning of milking installations for ewes in the region of Roquefort. Adapting the semi-automatic, acidified boiling water treatment to the milking installation "system Casse"

Roquefort cheese is made with raw ewe's milk. Therefore, the cleaning of the milking installation for ewes must be satisfactory ; more especially as their number is increasing yearly.

Firstly, all the surveys carried out at the experimental station "Casse » of the Société des Caves de Roquefort are reviewed.

From 1967 to 1970 , the experiments illustrate the connection between the quality of raw milk and the technique of cleaning the installation, the quality of the rinse water, the existence of latent mastitis, the cleaning of clusters, the atmosphere of the milking parlours, etc. 
The acidified boiling-water system (tested at N.I.R.D., England), was adapted to the Casse system in 1968. The cleaning assays, the assessments of the efficiency of the cleaning technique and the results on milk quality are described.

This technique is well adapted and efficient. It has been developed further since. The milk collected by the 4 milking installations cleaned with this system has been followed up since 1969. The quality level is excellent.

Moreover, the system is simple and practical and 3 times quicker than the usual cleaning techniques.

Reçu pour publication en septembre 1971.

\section{Bibliographie}

[1] Auclair (J.) et Richard (J.) (1966). - Traite mécanique et qualité du lait. Ann. de la Nutrition et de l'Alimentation, vol. XX, n ${ }^{\circ}$ 5, 29-41.

[2] Bosc (J.) (1966). - Progrès récents et problèmes de la traite mécanique des brebis. Ann. de la Nutrition et de l'Alimentation, vol. $\mathrm{XX}, \mathrm{n}^{\circ} 6$, 193-230.

[3] Clough, AKam et Cant (1969). - Méthode de nettoyage des machines à traire par circulation d'eau bouillante. N.I.R.D. paper, $\mathrm{n}^{\circ} 2,939,1-8$.

[4] DaRRACQ (1969). - Le nettoyage en circuit fermé des installations de traite. Agri. 7 jours, 3, (1), 15.

[5] Hunter (A.) and JeFFrey (D. C.) (1970). - Acidified boiling water treatment of pipeline milking machines. J. Soc. Dairy Technol, 23, (2), 75-77.

[6] JefFrey (D. C.) and Hunter (A.) (1970). - Acidified boiling water treatment of parlour milking installations. J. Soc. Dairy Technol, 23, (2), 70-74.

[7] Palmer (J.) and Cowhig (M. J.) (1968), - New method of cleaning bucket milking machines (boiling water). Farmer Resarch News, 9, (2), 40-41.

[8] RichaRd (J.) et Auclair (J.) (1962). - Comparaison de deux méthodes de nettoyage des machines à traire. Le Lait, janvier-février.

[9] Richard (J.) et Auclair (J.) (1966). - Essai d'un stérilisateur à vapeur pour la désinfection des manchons trayeurs. Revue Laitière Française, $\mathrm{n}^{\circ}$ 235, septembre, 502-505.

[10] RichaRd (J.) (1970). - Le nettoyage des installations de traite à l'eau chaude acidifiée. Producteur Agricole Français, $\mathrm{n}^{\circ} 78$, octobre.

[11] Société Anonyme des Caves et des Producteurs RÉunis de Roguefort (Aveyron). Traite mécanique des brebis, "système Casse ". $2^{\text {e }}$ édition 1967. 